\title{
Hepatitis Prolongadas y Crónicas en Niños Tratamiento y Evolución de 10 Casos
}

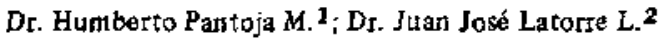

\author{
Protracted and Chronic Hepatitis in Children
}

Ten cases protracted hepatitis with laboratory and histological evidence are analized; Age fluctuated between 1 and $1210 / 12$ years of age, six patients were females, nine were treated with prednisone according to the scheme proposed by Arasu. The duration of treatment varied from 1/12 to $26 / 12$ years. Total remission was observed in seven patients. Difficulties in the diagnosis of $\mathrm{CAH}$ in children and in its differenciation from acute hepatitis with histological signs of chronicity are enphasized. Our results seems to justify the international recomendations to avoid performing hepatic biopsy before six months of evolution.

La hepatitis crónica $(\mathrm{HC})$ es una entidad cuya controvertida definición incluye características clínicas, de laboratorio $y$ morfológicas, todas ellas enmarcadas en un concepto de tiempo fijado por un Comité Internacional en 6 meses de inflamación continua del hígado, excluyendo el componente inflamatorio de la obstrucción crónica biliar y la producida por el alcohol. No obstante se han propuesto plazos que van de 3 a 4 semanas $^{1}, 10$ semanas $^{2}, 13$ semanas $^{4}, 3$ me$\operatorname{ses}^{4}, 4$ meses $^{5}$ y 6 meses 6 lo que ha llevado a que las diferencias casuísticas no sean del todo comparables entre sí, siendo esto más evidente en la literatura pediátrica.

En nuestro medio se ha propuesto el estudio histológico del higado cuando la hepatitis se prolonga por más de tres meses con persistencia de las transaminasas elevadas o su tendencia al aumento progresivo, prolongación del tiempo de protrombina, hiperganmaglobulinemia e hiperbilirrubinemia; además síntomas como anorexia, decaimiente, hepatomegalia, trastornos de coagulación y por complejo inmune.

Desde el punto de vista morfológico la diferenciación de las variantes de la hepatitis aguda y crónica es importante por las implicaciones pro. nósticas y terapéuticas, sin embargo no siempre

1 Unidad de Gastroenterología [nfantil Hospital Sótero del Río.

2 Servicio de Anatomía Patológica Hospital L. Calvo Mackenna. es posible mediante la biopsia percutánea diferenciar una hepatitis aguda de una crónica ${ }^{7}$ pudiendo verse sumados a la necrosis focal, necrosis fragmentaria y puentes de necrosis porto-portales, que a pesar de la severidad histológica van a la completa restauración sin dejar secuelas ${ }^{8}$ - $10-11$. También se ha descrito la dificultad para diferenciar la hepatitis no-A no-B aguda que en ocasiones se prolonga por más de 6 meses, de la hepatitis crónica producida por el mismo agente.

De Groote y col. ${ }^{22}$ han propuesto una subdivision de las $\mathrm{HC}$ en Hepatitis Crónica Persistente (HCP) y Hepatitis Crónica Activa (HCA) que es ampliamente aceptada.

La HCP es una forma benigna que se presenta con sintomas inespecíficos que eventualmente siguen a una hepatitis aguda. Histológicamente hay un infiltrado inflamatorio portal constituido por linfocitos y plasmacélulas, la arquitectura está conservada. Ocasionalmente puede haber necrosis fragmentaria y presentar exacervaciones agudas con necrosis confluente ${ }^{7}$. En general el pronóstico es bueno aunque se ha descrito la transición de $\mathrm{HCP}$ a $\mathrm{HCA}^{13-14}$. Este cuadro histológico no es específico y nosotros los hemos visto en relación a enfermedades sistémicas como la leucemia.

La HCA presenta un cuadro diferente con un infilltrado inflamatorio portal y periportal denso, con pérdida de la delimitante portolobulillar, fenómenos de necrosis fragmentaria y fibrosis. Puede haber también necrosis parcelar, necrosis en puente y formación de rosetas en la interfase 
de tejido conectivo y parénquíma. Estas lesiones pueden disminuir espontáneamente, 0 , más frecuentemente evolucionar hacia la cirrosis ${ }^{15}$ a no mediar el tratamiento esteroidal que en series controladas ha demostrado su eficacia en el manejo de la $\mathrm{HCA}^{16-17-18-19}$.

Parece haber consenso en que la $\mathrm{HCA}$ en el niño tiene mejor pronóstico que en el adulto. Ello puede explicarse por mayor poder de regeneración, mejor respuesta al tratamiento esteroidal, y muy probablemente sobrediagnóstico de la enfermedad, consecuencia de la tendencia a tomar muy precozmente la biopsia hepática en la mayoría de las series pediátricas, lo que lleva a catalogar HA con lesiones sobreimpuestas de cronicidad como HCA. Tampoco hay series pe. diátricas de control que permitan evaluar la evolución espontánea de la HCA como se ha hecho en adultos.

\section{MATERIAL Y METODOS}

Presentamos 10 niños en quienes se hizo el diagnóstico clínjco e histológico de HCA, cuyas edades fluctuaban al momento del diagnóstico entre 1 y 12 años 10 meses, con un promedio de 6 años 3 meses, 6 niñas y 4 varones, controlados por un tiempo de 1 a 5 años 10 meses con una media de 2 años. En ellos se midieron transaminasa glutámico pirúvica en el suero; bilirrubine- mia total y directa; tiempo de protrombina, electroforesis de proteinas plasmáticas, anticuerpos an tinucleares (AAN); Antígeno de superficies para hepatitis B (HBsAg) por método de Raphadex-B (inmunodifusión pasiva reversa) y en dos casos por Radioinmuno ensayo (RIA). En algunos niños se determinaron alfa-feto-prote ínas; gammaglutamil transferasa, anticuerpos anti-DNA; células de Lupus; ceruloplasmina y alfa-1-antitripsina. En todos se hicieron por lo menos dos biopsias por punción tomadas con aguja de Menghini 1,2 mm., fijadas en Bouin. En un caso la primera biopsia se hizo por laparotomia. Las muestras se tiojeron con HematoxilinaEosina, Van Gieson, PAS y técnica para reticulina, siendo informadas por uno de nosotros (JJ.L.), salvo dos derivadas de otros hospitales para su control en nuestro servicio.

Con la excepción del Caso 1 todos los demás pacientes recibieron tratamiento esteroidal diario, en las dosis habituales, hasta obtener un descenso de las transaminasas a 100 Uds. $x 1$., a partir del cual se suministró en días alternos, siguiendo el esquema propuesto por Arasu y $\mathrm{Col}^{20}$. La evolución fue controlada con mediciones de transaminasas, tiempo de protrombina y electroforesis de proteinas. La segunda biopsia se hizo entre tres y once meses después de la primera con una media de 5,8 meses.

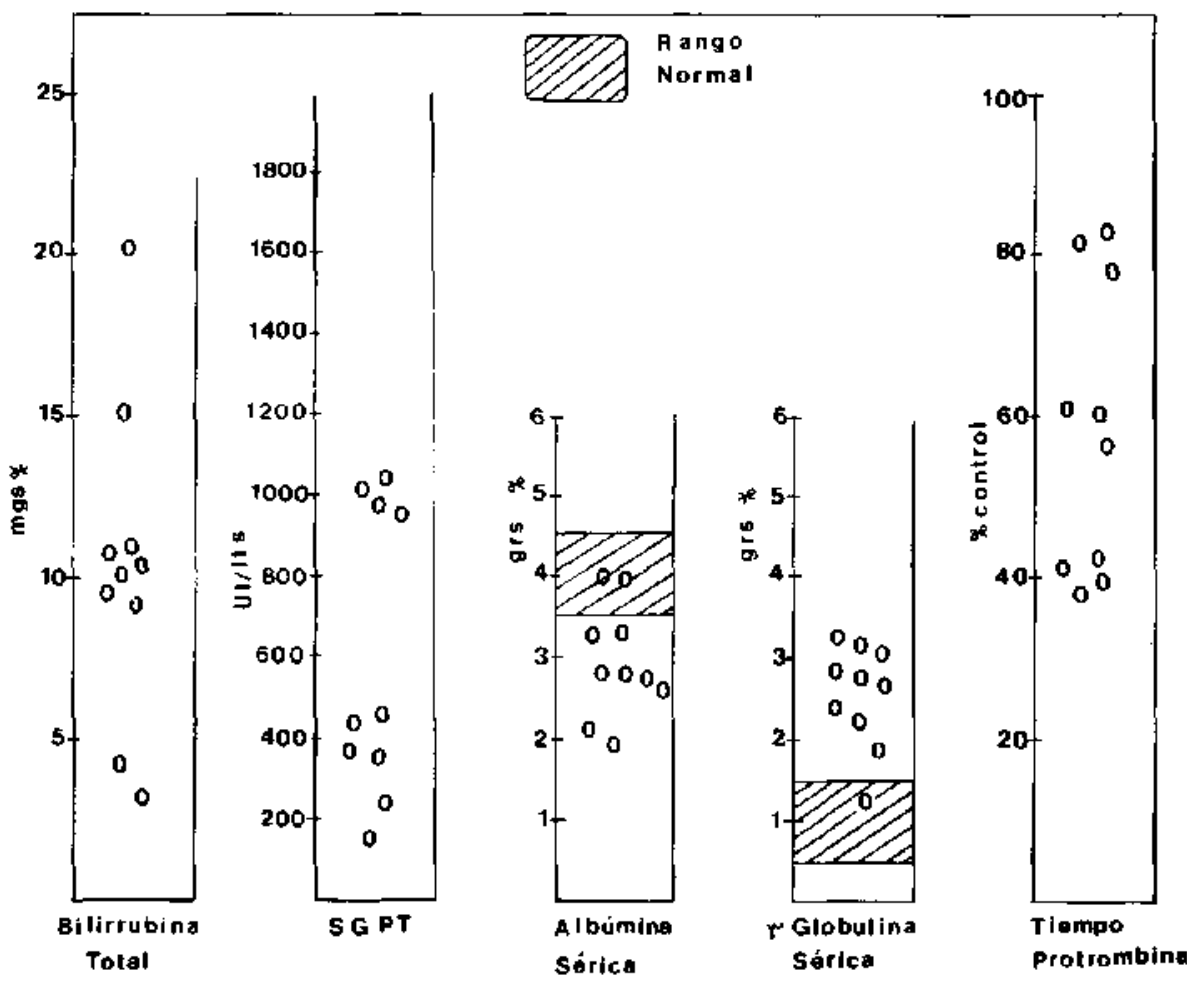

Figura 1.: parámetros bioquímicos en la sangre de 10 nimos con Hepatitis prolongada al comienzo de los síntomas. 
Tabla 1: Aigunos parámetros clínicos y duración del tratamiento en 10 niñas con H.C.A.

\begin{tabular}{|c|c|c|c|c|c|c|c|c|c|c|}
\hline CASO & EDA D & SEXO & $\begin{array}{l}\text { Tpa. } \\
\text { Proplo } \\
\text { Ia Giopaia }\end{array}$ & $\begin{array}{l}N^{0} \text { do } \\
\text { Blopsiats }\end{array}$ & $\begin{array}{c}\text { Marcadoras } \\
\text { de } \\
\text { Mepatiols }\end{array}$ & A.AN, & $\begin{array}{l}\text { Ceruleplas } \\
\text { mino }\end{array}$ & $\begin{array}{r}\text { Tpa. con } \\
\text { predrinona }\end{array}$ & $\begin{array}{l}\text { Tpo. on } \\
\text { conitol }\end{array}$ & $\begin{array}{l}\text { Lapso enlre } \\
1^{\text {era }} \times 2^{\text {do }} \\
\text { gloptit }\end{array}$ \\
\hline 1. MOF. & $11 \mathrm{a}$ & $\mathbf{F}$ & $2 \mathrm{~ms}$ & 2 & Heseg $(-)$ & - & $\mathbf{N}$ & 0 & $12 \mathrm{~ms}$ & $11 \mathrm{~ms}$ \\
\hline 2. Y.R.A. & $404 \mathrm{~m}$ & $\mathbf{F}$ & $3 \mathrm{~ms}$ & 2 & HBCA $(-)$ & - & M & $10 \mathrm{~ms}$ & $2 a$ & $7 \mathrm{~ms}$ \\
\hline 3. E.GC. & $12.10 \mathrm{~m}$ & $\mathbf{F}$ & $1 \mathrm{~m} v$ & 2 & HBQ Ay $(\cdot)$ & - & $\mathbf{N}$ & $5 \mathrm{~ms}$ & $134 \mathrm{~ms}$ & $4 \mathrm{~ms}$ \\
\hline 4. Yc. & $2 n \mathrm{~m}$ & $\mathbf{F}$ & $4 \mathrm{~min}$ & 2 & $H B_{B} \wedge_{8}(-)$ & - & - & $12 \mathrm{~ms}$ & 5 a $10 \mathrm{me}$ & $7 \mathrm{~ms}$ \\
\hline S. KC.A. & $6.1 \mathrm{~m}$ & $\mathbf{F}$ & $12 \mathrm{~ms}$ & 2 & $H B A_{g}(-)$ & $m$ & $N$ & $236 \mathrm{mc}$ & $286 \mathrm{~ms}$ & $\Delta \mathrm{m}$ \\
\hline B. A.A.A. & $7 \mathrm{2} \mathrm{m}$ & $\mathbf{F}$ & $4 \mathrm{~ms}$ & 3 & $H \in A_{Q}()$. & - & $\mathbf{N}$ & $8 \mathrm{~ms}$ & $204 \mathrm{~ms}$ & $4 m s$ \\
\hline 7. c.vo. & $1 \mathrm{a}$ & $M$ & $9 \mathrm{~ms}$ & 2 & $H B S A g(-1)$ & - & - & $5 \mathrm{mg}$ & $1.2 m A$ & $5 \mathrm{~ms}$ \\
\hline B.CA-Cu & $5.6 \mathrm{~m}$ & $\mathbf{m}$ & $11 \mathrm{~ms}$ & 4 & 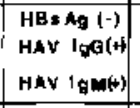 & - & 一 & $17 \mathrm{~ms}$ & $2 \mathrm{a} 3 \mathrm{~ms}$ & $3 \mathrm{ma}$ \\
\hline 9. F,C, T. & Q a $10 \mathrm{~m}$ & $M$ & $4 \mathrm{ma}$ & 2 & $\begin{array}{l}\text { HBEAg }(-1) \\
\text { antl } \mathrm{HBC}(-1) \\
\text { MAV I OMN }\end{array}$ & - & - & $8 \mathrm{mo}$ & $11 \mathrm{~ms}$ & $4 \mathrm{ma}$ \\
\hline 10. J.D.L. & $3.6 \mathrm{~m}$ & 4 & $2 / 2 \mathrm{~ms}$ & 2 & Hes A g(-) & - & $\mathbf{N}$ & $9 \mathrm{~ms}$ & $106 \mathrm{~ms}$ & $5 \mathrm{~ms}$ \\
\hline
\end{tabular}

\section{Caso 1.:}

Niña de 11 años de edad. En Septiembre de 1982 presentó signos de hepatitis con transaminasas de $545 \mathrm{U} / 1$ y bilimubinemia total de 5,64 $\mathrm{mg} \%$. Después de un mes de reposo las transaminasas fueron de $56 \mathrm{U} / 1$. A los dos meses de evolución reaparecieron el decaimiento y la ictericia, la bilirnubina total era de $13,16 \mathrm{mg} \%$, las fostatasas alcalinas $149 \mathrm{UI} / 1$ y las transaminasas de $250 \mathrm{U} / 1$, AAN, alfa-feto-proteinas y $\mathrm{HBsAg}$ negativos; electroforésis de proteínas con albúmina de $2,7 \mathrm{~g} / \%$ y gammaglobulina de $3.29 \mathrm{~g} \%$. La biopsia hepática a los $21 / 2$ meses de evolución fue informada como evidencia de hepatitis aguda con signos histológicos de cronicidad. Tratada sin prednisona y controlada periódicamente con transaminasas, protrornbinemia y electroforésis de proteínas (EFP) demoró 6 meses desde el comienzo de su enfermedad hasta la normalización de los parámetros nzencionados. La biopsia de control realizada 11 meses después de la primera mostraba alteraciones residuales de hepatitis.

\section{Caso 2:}

Niña de 4 afios 4 meses con signos de hepatitis de tres meses de evolución con bilirrubina total de $2,5 \mathrm{mg} \%$; transaminasas de $1.040 \mathrm{U} / 1$ gammaglobulina de $2,8 \mathrm{~g} \%$; protrombina de $54 \% ; \mathrm{HBsAg}$ negativo, alfa-1-antitripsina $315 \mathrm{mg} \%$; ceruloplasmina de $94 \mathrm{mg} \%$. La biopsia fue interpretada corno HCA por lo que se indicó prednisona en dosis decrecientes y luego en dís alternados, hasta que la EFP que fue normal y las transaminasas llegaron a $17 \mathrm{U} / 1$ por lo que se redujo la dosis a 5 mgs. en días alternados. La segunda biopsia se hizo a los 7 meses de la primera y fue informada con cambios residuales de hepatitis. La prednisona se suspendió tres meses después. Se controló durante dos años sin sufrir recaidas.

\section{Caso 3.:}

Niña de 12 años 10 meses. En Mayo de 1980 presentó síntomas de hepatitis con transaminasas de $950 \mathrm{U} / 1$, hiperbilirrubinemia persistente alta con cifras de hasta $20,3 \mathrm{mg} \%$, protrombinemia de $42 \%$, AAN y HBsAg negativos, ceruloplasmina de $58.3 \mathrm{mg} \%$, células LE negativas, EFP con gammaglobulina de $2,8 \mathrm{~g} \%$ y albuimina de $2,7 \mathrm{~g} \%$. La biopsia al mes de evolución clínica fue informada como $\mathrm{HCA}$ por lo que se dejó prednisona según 
e] esquema descrito. La segunda biopsia 4 meses después fue interpretada como hepatitis aguda con elementos de cronicidad. Se disminuyeron gradualmente los corticoides y controló periódicamente, normalizándose las transaminasas y las gammaglobulinas a los 5 meses de evolución por lo que se suspendió la prednisona controlándose por 16 meses sin tener recaidas.

\section{Caso 4:}

Niña de 2 años 6 meses con hepatitis comprobada por laboratorio 4 meses antes, ingresó por reaparición de la ictericia, bilirrubina total de $12,23 \mathrm{mg} \%$, transaminasas de $1.080 \mathrm{~L} / 1$, protrombinemia de $58 \%$ AAN y HBsAg negativos, EFP con gammaglobulina de $2,48 \mathrm{~g} \%$ y albúmina de $3 \mathrm{~g} \%$. La biopsia hepática fue informada como HCA. Se dio prednisona diaria en las dosis habituales. La nueva biopsia 7 meses después fue informada con cambios residuales de hepatitis. Se mantuvo los esteroides por un año, perdiéndose la niña de control durante 5 años y 2 meses al cabo de los cuales volvió con pruebas de laboratorio normales.

\section{Caso 5.:}

Niña de 6 años 1 mes. Desde Mayo de 1980 con síntomas de hepatitis y transaminasas de 525 y $720 \mathrm{U} / 1$ que se mantuvieron elevadas durante un año al cabo del cual se envía para su estudio: Tiempo de protrombina $60 \%$, transaminasas 400 $\mathrm{U} / 1$, bilirrubina total $11,9 \mathrm{mg} \%$, ceruloplasmina $61,3 \mathrm{mg} \%$, AAN y HBsAg negativos, gammaglobulina $2,84 \mathrm{~g} \%$. En la cintigrafía hepática aparecía hepatomegalia a expensas del lóbulo izquierdo y defectos de captación del sulfocoloide radiactivo. Se intentó la biopsia por punción en dos oportunidades sin obtener muestra por lo que se hizo biopsia quirúrgica en cuña que fue informada como cirrosis macronodular con fe. nómenos de necrosis fragmentaria e infiltrado plasmocitario. Se indicó prednisona $2 \mathrm{mg} \mathrm{x} \mathrm{kg} \mathrm{de}$ peso al día disminuyendo la dosis gradualmente hasta llegar a $15 \mathrm{mg}$. diarios. La segunda biopsia, por punción, fue interpretada como HCA moderada a severa con escasa proliferación de tejido conjuntivo. La concentración de transaminasas séricas más reciente era de 21,5 U/1 con EFP normal. Cumplió $21 / 2$ af́os en control sin signos de descompensación de la cirrosis.

\section{Caso 6.:}

Niña de 7 años 2 meses, comenzó en Marzo de 1980 con signos de hepatitis y transaminasas de $300 \mathrm{U} / 1$. Dos meses después del inicio de su afección seguía con transaminasas elevadas en $375 \mathrm{U} / 1$, tiempo de protrombina $62 \%$, albúmina plasmática $3,48 \mathrm{gr} \%$, gammaglobulina $2,28 \mathrm{~g} \%$, AAN y HBsAg negativos, ceruloplasmina de 68 mg\%. La biopsia hecha a los 4 meses de evolución fue informada como HCA. Tratada con prednisona según el esquema descrito, un mes después las transaminasas eran de $97 \mathrm{U} / 1$, por lo que se redujo la dosis de prednisona y se dejó en días alternados. 4 meses más tarde seguía con $10 \mathrm{mg}$ dia por medio y la segunda biopsia fue informada como secuelas de hepatitis. Controlada con tercera biopsia a los 9 meses de la primera se encontraron signos residuales de hepatitis. Desde entonces sin prednisona, ha sido controlada por dos antos y cuatro meses sin recaídas.

\section{Caso 7.:}

Niño de tres meses 15 días. Antecedente de ictericia desde el nacimiento. Transaminasas 180 $\mathrm{U} / 1$; bilirrubina total $8,94 \mathrm{mg} \%$, directa 3,96 $\mathrm{mg} \%$; tiempo protrombina $10 \%$, que sube a $100 \%$ después de tres días de tratamiento con vitamina $\mathrm{K}$; gammaglutamil transferasa $127 \mathrm{U} / 1$; alfafetoproteínas negativas; alfa-1-antitripsina 268 mg\%; Albúmina plasmática $2,87 \mathrm{~g} \%$, gammaglo. bulina $1,8 \mathrm{~g} \%$, HBsAg, VDRL y reacciones para toxoplasma y Chagas negativas. La primera biopsia se hizo a los tres y medio meses de vida y fue informada como hepatitis neonatal. En con. troles sucesivos, la ganmaglutamil transferasa se mantuvo en $97 \mathrm{U} / 1$, las transaminasas en $58 \mathrm{U} / 1$, la bilirrubina subió a $23 \mathrm{mg} \%$ total con directa $11,52 \mathrm{mg} \%$. Al año de vida se realizó nueva biopsia, que fue interpretada como $\mathrm{HCA}$ con gran proliferación de tejido conectivo. Se indicó prednisona, ha completado 5 meses de tratamiento continuo y actualmente las transaminasas son del orden de $72,5 \mathrm{mg} \%$, fosfatasas alcalinas 291 UI y bilirrubina total $12 \mathrm{mg} \%$ con directa de 6,5 $\mathrm{mg} \%$.

\section{Caso 8.:}

Niño de 5 años 6 meses. Un mes antes del ingreso presentó hepatitis con transamirasas de $2,700 \mathrm{U} / 1$ y bilirrubinemia total de $24,8 \mathrm{mg} \%$. Se mantuvo en reposo en su casa, notando los padres, cambios en los hábitos del sueño $y$ alteraciones conductuales, las transaminasas seguían altas y la protrombina llegó a $25 \%$ por lo que se hospitalizó. A su ingreso el higado se palpaba $5 \mathrm{~cm}$. bajo el reborde costal. Examen neurológico normal. Transaminasas séricas 1.900 U/1. El tiempo de protrombina no mejoró a pesar de las dosis repetidas de vitamina $\mathrm{K}$. Tratado con régimen hipoprotéico, enemas evacuantes diarios, y neomicina, cuantro días después el hígado no se palpa, cayó en sopor y debió ser trasladado a la Unidad de Cuidado Intensivo 
donde se dejó con régimen 0, cimetidina, cortisol, neomicina y controles de protrombina, bilirrs. bina, glicemia, uremia y Astrup. En pocas horas pasó a coma grado II a IIl, por lo que se efectuaron tres ex-sanguineo-transfusiones tomando previamente sangre para investigar marcadores de hepatitis. Entre el segundo y tercer recambio se tomó una biopsia hepática; dio como resultado una necrosis hepática difusa. Evolucionó favorablemente y fue dado de alta a los 23 días de hospitalización. Los marcadores de hepatitis por RIA mostraron HBsAg negativo, anti-HBc negativo, anticuerpos anti-HAV de tipo lgM negativos y anti-HAV de tipo IgG positivo, de manera que se interpretó como una hepatitis fulminante por vinus no-A no-B. Ha sido controlado durante 19 meses desde el comienzo de su enfermedad sin que las transaminasas se normalicen. La segunda biopsia tres meses después de la primera mostró HCP. Al mismo tiempo la concentración de garnmaglobulina en el plasma era de $2,2 \mathrm{~g} \%$; transaminasas $271 \mathrm{U} / 1$. La tercera biopsia se hizo a los 9 meses de la primera y mostraba una HCA por to que se dejó tratamiento con prednisona que provocó un síndrome de Cushing. Actualmente recibe $15 \mathrm{mg}$ diarios. La última biopsia se tomó 1 año 7 meses del inicio de su enfermedad y muestra evidencia de HCA. La EFP y la cintigrafia hepática son normales pero las transaminasas continúan sobre 100 $\mathrm{U} / \mathbf{1}$.

\section{Caso 9.:}

Nifío de 9 afios 10 meses. En Septiembre de 1982 comenzó con manifestaciones de hepatitis. Tres y medio meses más tarde tuvo dolor abdominal, vómitos y compromiso del estado general. A su ingreso los exámenes de laboratorio mostraban una bilirrubinemia total de $2,3 \mathrm{mg} \%$, transaminasas $980 \mathrm{U} / 1$; protrombina $80 \%$; Alfa-fetoproteínas negativas; gammaglutamil tranferasa 80 U/1; AAN negativos. A los 4 meses de evolución se hizo biopsia hepática por punción, cuyo resultado fue informado como HCA y se dejó en tratamiento esteroidal. En coincidencia con éste disminuyeron rápidamente las transaminasas a 25 $\mathrm{U} / 1$, por lo que se redujo la dosis de corticoides y se dejan día por medio. Cuando llevaba tres meses de tratamiento y siete de evolución llegaron los resultados de los marcadores de hepatitis tomados al ingreso, con $\mathrm{HBsAg}$ negativo, anti-HBc negativo, anticuerpos anti-HAV de tipo IgG negativos y anti-HAV de tipo lgM positivo lo que se interpreta como evidencia de hepatitis viral aguda por virus $A$. La prednisona se suspendió a los 6 meses de tratamiento. La segunda biopsia tomada 4 meses de la primera mostró signos residuales de hepatitis. Completó 11 meses de control sin problemas.

\section{Caso 10:}

Niño de 3 años 5 meses que en Enero de 1981 presentó evidencia de hepatitis con transáminasas $380 \mathrm{U} / 1$, bilirrubina total $8 \mathrm{mg} \%$, protrombina 60\%. En Marzo del mismo afto se agregó un exantema máculopapular del tronco y extremi. dades, hepatomegalia, espienomegalia y artralgias de rodillas; AAN y HBsAg negativos; anticuerpos anti-DNA con $14 \%$ de enlace; Albúmina $3,96 \mathrm{~g} \%$ y gammaglobulina de $2,37 \mathrm{~g} \%$ en el plasma, IgG $2.500 \mathrm{mg} \%$, IgM $250 \mathrm{mg} \%$; ceruloplasmina 60 $\mathrm{mg} \%$; la biopsia hepática a los 2 1/2 meses de evolución fue informada como HCA. Se dio prednisona en las dosis corrientes desapareciendo las lesiones cutáneas. Recibio esteroides por algo más de un mes, perdiéndose del control durante 7 meses al cabo de los cuales tenía transaminasas, protrombina, proteinas plasmáticas y biopsia hepática normales. Luego se controló durante 18 meses sin suftir recaidas.

\section{COMENTARIO}

Los casos presentados no son exactamente comparables entre sí pero permiten obtener algunas conclusiones. Es indudable que el Caso 1 es una hepatitis aguda en que el estudio histológico no sirvió para tomar una decisiớn, y aún cuándo la biopsia de control 11 meses después muestra cambios residuales es improbable que evolucione a la cronicidad. Seguramente los Casos 2, 3, 4, y 6 hubieran evolucionado hacia la remisión total aún sin tratamiento con prednisona, como ocutrió en el Caso 1. Si en el Caso 9 se hubiesen tenido los marcadores más precozmente el niño se habrja ahorrado la biopsia y el tratamiento esteroidal pues no se ha descrito HCA por virus $A$, pero este caso nos enseña que la hepatitis $A$ puede dar lesiones histológicas importantes con sobreposición de alteraciones crónicas sobre una hepatitis aguda y prolongarse por meses antes de la remisión total.

El caso 10 fue diferente porque presentó una Acrodermatitis Papular que ha sido relacionada con hepatitis $\mathrm{B}^{21}$, aunque recientemente tam. bién con hepatitis por virus no-A no- $\mathrm{B}^{\mathbf{2 2}}$ y por virus de Epstein-Barr ${ }^{23}$. Desgraciadamente no se descartó la posibilidad de Mononucleosis infec. ciosa ni de hepatitis no-A no-B. La evolución posterior con completa remisión hacen pensar en una hepatitis por virus Ebstein-Barr o no-A no-B.

El Caso 5 pese a la tardía referencia desde el 
consultorio externo ha evolucionado bien, no ha tenido signos de sindrome de Cushing y la cirrosis aparentemente no ha avanzado.

El Caso 7 escapa a lo habitual en las hepatitis neonatales ya que hay remisión total o rápida evohución a la cirrosis biliar. El tratamiento con prednisona no ha significado mejoría evidente del nif̃o y su pronóstico pudiese estar determinado por su colestasis crónica más que por las manifestaciones de HCA sobrepuesta, pese a lo cual decidimos mantenerlo con prednisona en dosis de $2 \mathrm{mg} / \mathrm{Kg}$ peso diario.

También es inhabitual el Caso 8, porque se ha descrito que las personas que sobreviven de ила hepatitis fulminante quedan sin daño hepático, siendo muy infrecuente la evolución hacia HCA o $\begin{array}{llll}\text { cirrosis }^{24} & -25 & -26 & -27\end{array}$. sin embargo por tratarse de una hepatitis no-A no-B podía preverse el curso arrastrado ${ }^{28}$ que sufrió. Sus probabilidades de llegar a la cirrosis son, sin embargo menores que si la infección hubiese sido por virus " $\mathrm{B}$ "20.

\section{CONCLUSIONES}

A ún cuando la biopsia hepática percutánea es muy útil en el diagnóstico de algunas enfermeda. des metabólicas y de depósito, y en el diagnóstico diferencial de las colestasis del primer trimestre de vida su rendimiento para diferenciar las hepatitis agudas con signos de cronicidad de las HCA es bajo. A menos que se conjuguen el tiempo de evolución, los marcadores de hepatitis, los anticuerpos anti-hísticos, el laboratorio general y la clínica, no servirá sino para seguir la evolución y la respuesta al tratamiento esteroidal. Es posible que si se respetan los plazos de 6 meses fijados por el Comité Internacional para efectuar la biopsia se reduzcan los errores de interpretación pero todavía queda el problema de las hepatitis no-A no-B que exceden dicho período de tiempo sin ser crónicas.

No hay acuerdo entre los gastroenterólogos infantiles sobre el tiempo que se debiera esperar para efectuar la biopsia hepática en una hepatitis que se escapa al curso habitual, por otro lado no siempre el comienzo de los síntomas marca el real comienzo de la enfermedad y ciertamente la primera manifestación puede ser una cirrosis florida. Zacarias, en nuestro medjo cree que estas hepatitis son realmente crónicas pero de mejor pronóstico que su similar del adulto. Los autores pensamos que siendo realmente de pronóstico favorable en la mayoría de los casos es preferible catalogarlas como hepatitis prolongada o de curso no habitual y esperar los plazos de 6 meses propuestos por el Comité Internacional para efectuar la biopsia si persisten los sintomas y las alteraciones de laboratorio.

\section{REFERENCIAS}

1 Mowat, Alex: Enfermedades hepáticas en la infancia. Editorial Pediátrica, Barcelona. A.P. Mouat, Pág. 209, 1980.

${ }^{2}$ Geal, M.G. et al: Classification and treatment of chronic active liver discase. Gastroenterology 55: $724,1968$.

${ }^{3}$ Nielsen, J.O. et al: Incidence and meaning of persistence of Australia antigen in patients with acute viral hepatitis: development of chronic hepatitis. New Engl. J. Med. 285: 1157, 1971.

4 Popper, H.; Schaffner, F.: The vocabulary of chronic hepatitis. New Engl. J. Med. 284; 1154, 1971.

5 Guiscafé, H. y col.: Hepatitis crónica en nimos. Bol. Med. Hosp. lnfant. Mex. 39: 399, 1982.

${ }^{6}$ Scherlock, S.: Chronic lepatitis. Gut 15: 581, 1974.

${ }^{T}$ Bianchi, L.: Liver Biopsy interpretation in hepatitis. Pat $\amalg$, Histopathology and classification of acute and chronic viral hepatitis. Differencial diagnosis. Path. Res. Pract. 178: 180, 1983.

8 Kerr, J.F.R. et ol: The nature of piecemeal necrosis in chronic active hepatitis. Lancet 2: 827, 1979.

9 Bianchi, L. et al: Acute and chronic hepatitis revisited. Lancet 2: $914,1977$.

10 Bianchi, L. et al: Morphological criteria in viral hepatitis. Lancet 1: 333, 1971.

11 Fauerholdt, L. et al: Significance of suspected "chronic aggressive hepatitis" in acute hepatitis. Gastroenterology 73: 543, 1977.

12 De groote, J. et al: A classification of chronic hepatitis. Lancet 2: 626, 1968.

13 Chadwick, R.G. et al: Chronic persistent hepatitis: hepatitis $B$ virus markers and histological follow-up. Gut 20:372, 1979.

14 Craja, A.J. et al: Corticosteroid-treated chronic active hepatitis in remission-uncertain prognosis of chronic persistent hepatitis. New Engl.J. Med. 304: 5,1981 .

15 De Croote, J. et al: Long-term follow-up of chronic active hepatitis of moderate severity. Gut $19: 510,1978$.

16 Cook Gc,i: Mulligan, R.; Scherlock, S.: Controlled prospective trial of corticosteroid therapy in active chronic hepatitis. Q.J. Med. 40: 159, 1971.

17 Solowoy, R.D. et al: Clinical, biochemical and histological remission of severe chronic active liver disease; a controlled study of treatments and early prognosis. Gastroenterology 63: 820, 1972.

18 Wright, E.C. et al: Treatment of chronic active hepatitis. Analysis of three controlled trials. Gastroenterology 73:1422, 1977.

19 Summerskill W.H.J. et al: Prednisone for chronic active liver disease: dose titration, standard dose, and combination with azathioprine compared. Gut 16: $876,1975$.

20 Arasu, t.S. et al: Management of chronic aggresive hepatitis in children and adolescents. J. Pediatr. 95: $514,1979$.

21 Gionotti, F.: Papular acrodematitis of childhood-an Australian antigen dise ase. Arch. Dis, Child: 48: 794, 1973.

22 pons Romero. F. et al: Hepatitis no-A no-B. Editorial Hepatologia y Gastroenterología 6: 32 , 1983.

23 Konno, M. et al: A possible association between hepatitis-B antigen-negative infantile papular acrodermatitis and Epstein-Barr virus infection. J. Pediatr. 101:222, 1982. 
24 Desmet, V.J. et al: Acute hepatocellular failure. A study of 17 patients treated with exchangetransfusion. Hum. Pathol 3: 167, 1972.

is Karvountzis, G.G.et al: Long-term follow-ip studies of patients surviving fulminat viral hepatitis. gastroenterology 67: 870, 1974 .

26 Hermier, $M$. et al: Hepatite chronique active d'evolutjon favorable, au decours d'une hepatite fulminante. Arch, franç. Pediat. 36: 865, 1979.
${ }^{27}$ Homey, J.T.; Galambos, J.: The liver during and after fulminat hepatitis. Gastroenterology 73:639, 1977.

28 Rakela, J., Redeker. A.: Chronic liver disease after acute non-A non-B viral hepatitis. Gastroentero$\operatorname{logy} 77: 1.200,1979$.

29 Krodell, R.g. et al.: Development of chronic disease after acute non-A non-B post-transfusion hepatitis. Gastroenterology 72: 902, 1977 . 